\title{
A vida após o acidente vascular cerebral na perspectiva dos sobreviventes
}

Jaine Kareny da Silva ${ }^{1}$, Vanessa da Silva Carvalho Vila ${ }^{2}$, Maysa Ferreira Martins Ribeiro ${ }^{3}$, Luc Vandenberghe ${ }^{4}$

${ }^{1}$ Enfermeira, Mestre em Ciências Ambientais e Saúde. Professora Assistente da Universidade do Estado da Bahia, Campus XII. Guanambi, BA, Brasil. E-mail: jksilva@uneb.br.

${ }^{2}$ Enfermeira, Doutora em Enfermagem. Professora Adjunta da Pontifícia Universidade Católica de Goiás (PUC Goiás). Goiânia, GO, Brasil. E-mail: vscvila@uol.com.br.

${ }^{3}$ Fisioterapeuta, Doutora em Ciências da Saúde. Professora Assistente da PUC Goiás. Goiânia, GO, Brasil. E-mail: maysafmr@yahoo.com.br.

${ }^{4}$ Psicólogo, Doutor em Psicologia. Professor Adjunto da PUC Goiás. Goiânia, GO, Brasil. E-mail:

luc.m.vandenberghe@gmail.com.

Recebido: 24/03/2015.

Aceito: 24/09/2015.

Publicado: 31/03/2016.

Como citar esse artigo:

Silva JK, Vila VSC, Ribeiro MFM, Vandenberghe L. A vida após o acidente vascular cerebral na perspectiva dos sobreviventes. Rev. Eletr. Enf. [Internet]. 2016 [acesso em:_____ Disponível em:

http://dx.doi.org/10.5216/ree.v18.34620.

\section{RESUMO}

Estudo de caso interpretativo desenvolvido com objetivo de compreender os significados da experiência da enfermidade na perspectiva de oito sobreviventes ao Acidente Vascular Cerebral. Os dados foram coletados por meio de entrevistas semiestruturadas, seguida de análise temática qualitativa. A experiência da enfermidade gerou sensações negativas como medo da morte, invalidez, perda de autonomia e incapacidade para o trabalho. O apoio social da família e da religião foi essencial para lidar com as mudanças na vida cotidiana na ineficiência das redes de atenção à saúde. Foi identificada a ausência de orientação, sobretudo pelos enfermeiros, para o cuidado do paciente no domicílio. Os resultados sugerem a necessidade de reforçar a educação em saúde para a valorização da sintomatologia preditora ao acidente vascular cerebral, a sensibilização do impacto que essa enfermidade representa na vida dos sobreviventes e a necessidade da equipe multiprofissional investir na proatividade dos familiares.

Descritores: Acidente Vascular Cerebral; Reabilitação; Pesquisa Qualitativa; Doença Crônica; Assistência Centrada no Paciente.

\section{INTRODUÇÃO}

O acidente vascular cerebral (AVC) consiste, mundialmente, na principal causa de morte e incapacidades em adultos ${ }^{(1-3)}$. É estimado que $74 \%$ das pessoas que sobrevivem ao AVC $57 \%$ necessitarão de cuidados dos familiares ${ }^{(4)}$.

Estudos apontam que apesar das sofisticadas tecnologias em saúde, a recuperação após o AVC é complexa e envolve aspectos biomédicos, sociais e psicológicos relacionados à saúde, bem-estar e qualidade 
de vida ${ }^{(5-6)}$. A maioria dos sobreviventes e familiares enfrentarão cotidianamente demandas de cuidados relacionadas ao comprometimento do nível de dependência e às necessidades complexas de cuidados, frente às disfunções físicas, cognitivas e emocionais decorrentes do $A V C^{(4-5,7)}$.

O AVC é descrito como um evento que, de modo súbito, muda a vida das pessoas e de seus familiares. As evidências científicas apontam a necessidade de que os sistemas de saúde estabeleçam intervenções que favoreçam a continuidade dos cuidados após a fase aguda com ênfase no período de reabilitação e reinserção social e comunitária ${ }^{(6-7)}$.

Assim, investigar o processo de reabilitação de pessoas que vivenciaram o AVC é importante, considerando-se sua elevada prevalência no cenário mundial e as repercussões na qualidade de vida das pessoas. Isso requer ações integradas de saúde que empoderem e capacitem as pessoas para alcançar e manter os melhores níveis de funcionalidade ${ }^{(6)}$.

Recente revisão sistemática sobre a sobrecarga vivenciada no tratamento do AVC, evidenciou que esse tratamento pode ser influenciado por aspectos relacionados aos níveis micro e macro dos sistemas de saúde e poderão variar entre as diferentes localidades. Como lacuna apontou-se a necessidade de compreender a experiência dos pacientes relacionado ao tratamento do AVC em diferentes contextos culturais ${ }^{(5)}$.

O desenvolvimento de estudos centrado nas pessoas é recomendado para compreender os desafios globais associados à continuidade do cuidado entre os serviços de saúde e o ambiente sociocultural vivenciado pelas pessoas. Essa compreensão pode ser importante para identificar a trajetória da enfermidade e os aspectos relacionados à gestão do cuidado em saúde frente ao AVC, contribuindo para gerar medidas de resultado centrado nas pessoas que serão utilizados por gestores da saúde pública e profissionais de saúde ${ }^{(5)}$.

Assim, o objetivo dessa pesquisa foi compreender os significados da experiência da enfermidade na perspectiva de pessoas que sobreviveram ao Acidente Vascular Cerebral.

\section{MÉTODO}

Estudo de caso interpretativo que focalizou a experiência da enfermidade na perspectiva de pessoas que sobreviveram ao AVC e foram atendidas em uma unidade hospitalar do Sistema Único de Saúde (SUS), do interior do nordeste brasileiro, de janeiro de 2011 a dezembro de 2012.

Os participantes foram as pessoas que atenderam aos seguintes critérios de inclusão: idade maior ou igual a 18 anos, diagnóstico médico de AVC e ser residente no município em estudo.

Inicialmente, realizou-se o levantamento dos potenciais participantes por meio dos prontuários localizados na unidade hospitalar e identificou-se que 37 pessoas que sobreviveram ao AVC atendiam aos critérios de inclusão. Por meio do contato telefônico foram registradas 28 exclusões relacionadas à mudança de cidade (14), déficit de comunicação (13) e óbito (1). Considerou-se perda a pessoa que não desejou participar da pesquisa (1).

Para definição dos participantes foi adotado o critério de amostragem intencional e saturação 
teórica ${ }^{(8)}$. O grupo social foi constituído por oito pessoas que sobreviveram ao AVC.

Os dados foram coletados de janeiro a dezembro de 2013, por meio de entrevistas semiestruturadas, individuais, face-a-face, em profundidade. Todas as entrevistas foram conduzidas no domicílio, a partir de um roteiro que contemplou as características socioeconômicas e as condições de saúde da pessoa que sobreviveu ao AVC. As questões norteadoras foram: O que aconteceu com você no dia do derrame? Como você se sentiu em relação a esta experiência? O que ajuda você a enfrentar este processo? Como ficou sua vida após o derrame? As entrevistas foram gravadas em áudio ou manuscritas, mediante o consentimento dos participantes e, posteriormente, transcritas na íntegra.

O número de entrevistas por participante foi definido no processo simultâneo de coleta e análise dos dados, considerando-se o momento em que o fenômeno fosse descrito em profundidade de modo fidedigno e aprofundado ${ }^{(8-9)}$. A saturação teórica foi obtida com a realização de 12 entrevistas, com a duração mínima de 30 minutos e máxima de 55 minutos. A realização de mais de uma entrevista para alguns participantes relacionou-se a necessidade de rever as lacunas da coleta, aprofundar e checar com cada participante a análise preliminar, para garantir o rigor metodológico em estudos qualitativos ${ }^{(9)}$.

A análise temática interpretativa foi conduzida em um processo contínuo e simultâneo a coleta de dados, por meio das seguintes etapas: familiarização com os dados, identificação dos códigos, agrupamento dos códigos em categorias temáticas, revisão das categorias, definição e nomeação final das categorias e elaboração da descrição e interpretação dos significados expressos pelos participantes em cada categoria ${ }^{(10)}$.

Para garantir a confiabilidade da análise dos $\operatorname{dados}^{(11)}$ foram utilizados três critérios: 1 - a gravação das entrevistas e a checagem das transcrições realizadas pelos dois primeiros autores do estudo; 2 - a confirmação, com os participantes, sobre as descrições ao final das entrevistas; 3 - os códigos e as categorias temáticas foram checados entre os membros da equipe de pesquisa ao longo do processo de análise dos dados. Todo o processo de interpretação foi conduzido coletivamente, por meio da discussão, revisão e reelaboração das descrições e interpretações.

O estudo foi aprovado pelo Comitê de Ética em Pesquisa da Pontifícia Universidade Católica de Goiás sob o protocolo no 305.390 e obedeceu aos aspectos éticos legais da legislação brasileira para pesquisa envolvendo seres humanos. Todos os participantes tiveram sua identidade protegida e assinaram o Termo de Consentimento Livre e Esclarecido (TCLE).

\section{RESULTADOS}

Participaram do estudo três homens e cinco mulheres, com média de idade de 54 anos, a maioria casados e com filhos. A maioria apresentou tempo de escolaridade menor que um ano e a renda mensal familiar entre um e dois salários mínimos. Apenas dois participantes retornaram ao trabalho após o AVC. Dois aposentaram, sendo um por invalidez definitiva e outro por idade. Os demais mencionaram dificuldades burocráticas para obter o direito da aposentadoria.

A análise interpretativa da experiência da enfermidade, vivenciada por pessoas que sobreviveram ao 
AVC, foi marcada por situações de fragilidade assistencial, sobretudo, no retorno ao domicílio. Todos os participantes descreveram que o AVC foi evento traumático e inesperado, que trouxe para suas vidas sentimentos de medo, desesperança e falta de preparo para reconhecer os sintomas e saber o que fazer.

A maioria dos participantes mencionou que já havia, em algum momento, sentido que algo estava estranho, mas pensavam que "na época era um detalhe e que iriam melhorar"! No dia do "derrame" os sintomas percebidos foram descritos como "a dor de cabeça", "o desvio da boca para um lado", "a conversa com dificuldade", "a convulsão" e "a dormência nos braços e nas pernas".

Essas pessoas encaminhadas com alterações clínicas à rede hospitalar vivenciaram fragilidades assistenciais como demora no atendimento, falta de pessoa qualificada para realizar exames importantes como a tomografia e segurança assistencial.

Após o atendimento, a maioria dos participantes retornou ao domicílio com alterações como a hemiparesia ou hemiplegia e tornou-se dependente de outras pessoas para realizar as atividades de vida diária. Expressaram o medo e a preocupação em tornarem-se dependentes para atividades mais simples, como a alimentação, assim como o receio de ocorrerem outras complicações no estado de saúde, como a morte. Esses sentimentos geraram desespero frente à incapacidade imposta pela enfermidade.

Por estar paralisada, a sensação foi de impotência! [...] aquele sentimento de que perdi tudo e não vou sair dessa! [...] Foi uma sensação de morte! A pior sensação [...] foi aquela! (P5).

Na minha cabeça eu pensava: [...] Será que eu vou ter que ficar assim para sempre? Direto? Dependendo? (P6).

Eu tenho vontade de fazer as coisas, que todo mundo faz para mim! [...] Arrumam a roupa, dão o banho, vestem e me dão o que comer! Eu fico lembrando que eu não precisava disso! [...] Tudo o que comer tem que ser bem cozido, bem machucado, por que, se ficar qualquer farelo na boca, não desce! [...] Se tomar água, eu fico com medo de engasgar! [...] À noite eu uso fralda porque eu não sento! Eu não quero acordar ninguém de noite para me apanhar e toda hora dá urina solta! [...] Eu sinto uma quentura! É muito ruim, mas é obrigado! Se fosse usar o urinol era melhor, mais folgado! (P4).

A falta do trabalho e/ou as limitações financeiras foram os problemas predominantes. $O$ impacto das incapacidades físicas transcenderam os cuidados das atividades cotidianas e repercutiram na situação econômica. A maioria dos participantes mencionou: "o medo de perder o benefício do INSS", "a interrupção das atividades", "a falta do trabalho", "o retorno às atividades lentamente", "a interrupção das atividades antes do horário habitual" e "a vontade de trabalhar".

Tenho vontade de trabalhar e não consigo! Sinto falta do trabalho! (P2).

Piorou a questão do trabalho! [...] eu ficava no bar até às duas horas da manhã [...] Hoje eu fecho e durmo mais cedo, por que eu tenho medo de voltar o derrame, fico tremendo! (P3).

Além da falta do trabalho, dois participantes também mencionaram que o "derrame" trouxe prejuízos na sociabilidade: "a vida ficou ruim", "sentem que atrapalham o lazer dos familiares" e "lamentam não poder mais viajar". 
Caminhava, viajava e hoje eu não posso! [...] por conta do AVC! [...] Se eu precisar ir à rua, ao banco e hospital, a menina [filha] precisa ir mais eu! Não posso sair só! E de primeiro eu fazia minhas coisas tudo só! Não dependia de ninguém! (P1).

Essas pessoas contaram com o apoio social de familiares para auxiliá-los ou realizar essas atividades por elas devido à privação imposta pela enfermidade. Essa experiência despertou sentimentos como: "medo de cair e não se curar", "nervosismo", "dependência", "perda da liberdade" e "tristeza".

O apoio social da família, assim como a crença em Deus e a espiritualidade, foram considerados importantes para superar essa etapa.

A presença dessas pessoas foi fundamental para a melhoria do quadro clínico e emocional dos participantes que descrevem que a ajuda ocorreu por meio de conversa, presença de amigos, passeios, atenção quanto aos pequenos desejos e na mudança no ambiente da casa para maior conforto.

Essa situação proporcionou o fortalecimento do vínculo familiar e acolhimento por meio da proximidade, cuidado e ampliação do amor entre o núcleo familiar. Segundo a maioria dos participantes, a fé em Deus foi determinante para superar a doença. Assim, descrevem que nesse momento de aflição "clamava ao Senhor que libertasse da doença", acreditavam que "Deus iria melhorar" aquela enfermidade e "sentia que Deus iria recuperar" a sua saúde.

Os participantes ainda contaram com o apoio formal dos profissionais das unidades de saúde. 0 principal recurso disponibilizado e usufruído foi a fisioterapia, presente na maioria das falas dos participantes, com avaliação positiva e negativa, seja no atendimento ou acesso ao serviço.

Foi muito tempo de fisioterapia que me ajudou! [...] O dia que eu fico, por exemplo, muito sentada, a minha perna e o braço ficam pior e se eu faço a fisioterapia melhora cem por cento! (P6).

Entretanto, um participante descreveu que, embora esse serviço fosse ofertado pelo município, existia uma dificuldade em realizar a marcação, mesmo com o pedido em mãos. Por essa razão algumas vezes teve que arcar com o pagamento por conta própria.

[...] Fez fisioterapia uma vez só aqui em casa [...] Tudo pelo hospital! [...] mas eles não vieram mais! [...]! (P4).

Outros serviços como a dispensação de medicação e marcação de exames, também foram mencionados por três participantes. Desses, dois participantes mencionam que conseguem ter acesso à medicação na unidade básica de saúde com facilidade, o que auxilia no controle da hipertensão. A demora na marcação de exames, mesmo o essencial como a tomografia, é apontado por dois participantes.

Se eu estou preocupada com a minha pressão, eu tenho o posto! [...]! Quando o meu remédio tá quase acabando, vou correndo para pegar! Até para gente pegar as receitas e passar no médico, que antes era difícil, hoje não! [...] O PSF é uma das coisas que facilitou muito para todo mundo! (P1).

Ele [o médico do PSF] fez o pedido da ultrassonografia Doppler, eletrocardiograma e da tomografia! Os dois primeiros eu marquei rápido [um mês], mas a tomografia só podia ser feito com relatório do neurologista! [...] demorou muito! [...] Três meses, mas tinha gente esperando há oito meses! (P6).

[no primeiro AVC] $O$ neurologista avaliou a tomografia e disse que com 48 horas era para fazer outra 
tomografia! [...] Eu tive alta, não foi feita a tomografia e fui encaminhada para marcar um neurologista pelo postinho! [...] Mas não conseguia vaga! (P8).

Os depoimentos acerca da vida após o AVC mostrou o quão impactante pode ser essa enfermidade na vida das pessoas. As restrições físicas causaram mudanças não só nas tarefas diárias, como também na autoestima, na capacidade para o trabalho e como consequência para a vida social e financeira. Apesar da dificuldade para a marcação de fisioterapia e acesso a outros recursos como a dispensação de medicação, esses suportes ainda são valorizados pelos participantes.

\section{DISCUSSÃO}

Por meio desse estudo, foi possível compreender a experiência intensa da enfermidade das pessoas que sobreviveram ao AVC pelas descrições das repercussões físicas, emocionais, sociais e econômicas. Descrever essas diferentes dimensões, interlaçadas, torna possível ver a complexidade das demandas de cuidado à saúde da fase aguda ao período de reabilitação de forma mais clara.

Os resultados apontaram a necessidade de sensibilizar o público para a adequada compreensão dos sinais e sintomas associados ao AVC de modo a efetivar a prevenção desse agravo. Além disso, observou-se a necessidade da educação popular para o reconhecimento precoce dos sinais e sintomas do AVC e a busca imediata de cuidados à saúde, pois o conhecimento inadequado tem prejudicado o prévio acesso aos cuidados disponíveis ${ }^{(12)}$.

Muitas pessoas tendem a subestimar os primeiros sinais e sintomas de enfermidades graves, o que interfere na busca do adequado enfrentamento do problema. O reconhecimento tardio desses sinais e sintomas compromete a chance de salvar a vida e, no caso de sobrevida, as chances de minimizar as incapacidades decorrentes dessa enfermidade ${ }^{(12-13)}$.

O bem-estar e a funcionalidade encontram-se comprometidos para essas pessoas, impactando na capacidade de desenvolver as atividades cotidianas ${ }^{(4,7)}$. Estima-se que na faixa etária superior a 50 anos, o AVC seja a causa de incapacidades que representa $40 \%$ das aposentadorias precoces e, mais além, cerca de $40 \%$ das mortes que ocorrem na América Latina, acontecem durante os anos mais produtivos da vida da pessoa $^{(14)}$.

As disfunções decorrentes do AVC geram dependência, sendo que em média $44 \%$ das pessoas não retornam ao trabalho após o episódio e necessitam de algum tipo de auxílio no desempenho de atividades cotidianas básicas $^{(15-16)}$. A perda de produtividade e a resistência para desenvolver as atividades laborais são notáveis. Frustrações e declínio no bem-estar subjetivo decorrem da perda de status e relações sociais ${ }^{(17-18)}$. Pesquisadores descrevem que retornar ao trabalho não é o único problema. Assegurar que o sobrevivente ao AVC mantenha-se no mercado de trabalho e a dificuldade de recurso financeiro e apoio social para manter o seu próprio tratamento também são importantes dificuldades vivenciadas por essas pessoas $^{(15)}$.

A incapacidade da pessoa que sofreu AVC para contribuir financeiramente gera sobrecarga para os 
familiares, que passam a assumir as despesas para suprir as necessidades de alimentação, vestimentas, aprimoramento do ambiente para as deficiências físicas, e arcar, sobretudo, com os gastos complementares à saúde, além de outras despesas não planejadas ${ }^{(5,7,13,18)}$.

A situação de dependência abala a estabilidade emocional e a própria identidade, suscitando sentimentos de inutilidade e fracasso. A pessoa sente-se triste, sem esperança e diminui o interesse nas atividades que eram prazerosas, retardando o processo de recuperação da saúde física e mental ${ }^{(4-5,7,19)}$.

Esses sentimentos oriundos da incapacidade funcional abalam ainda mais o convívio social, gerando

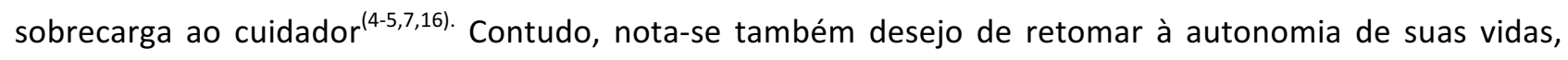
quando amparadas por um suporte social formal ou informal ${ }^{(17,19)}$.

O apoio social da família e o conforto espiritual foram estratégias importantes para o enfrentamento e podem ter sido as forças propulsoras para o restabelecimento da saúde de alguns pacientes, perante a precariedade das unidades de saúde para a garantia da continuidade dos cuidados em domicílio.

As evidências científicas apontam que quanto mais suporte social essas pessoas receberem, melhor será a percepção de qualidade de vida e melhores serão os resultados relacionados à capacidade do indivíduo para enfrentar e adaptar-se as mudanças em sua vida diária decorrentes do $\mathrm{AVC}^{(20-21)}$.

A religião e a espiritualidade apoiam a esperança de melhorias que podem ser alcançadas pela fé. As atividades religiosas geralmente melhoram a qualidade de vida e o propósito de viver. Dessa forma, a religião tem sido associada positivamente com melhor saúde física e mental após o $\mathrm{AVC}^{(15,22)}$. Nesse contexto, a integração da família e fé em Deus foram componentes chaves para o suporte emocional e para melhorar a capacidade de enfrentamento da enfermidade ${ }^{(20-21)}$.

A precariedade assistencial se mostrou, sobretudo, no momento de orientações relacionadas a continuidade dos cuidados no contexto domiciliar e comunitário, que deveriam ser iniciados no ambiente hospitalar ${ }^{(4,6,19)}$. Verificou-se que o modelo de cuidado vigente ainda não incorporou as diretrizes relacionadas a reabilitação e a promoção da saúde com vistas a adaptação e a manutenção do bem-estar e funcionalidade ${ }^{(21)}$, centrando-se, ainda, na cura da enfermidade e não no cuidado das pessoas e seus familiares, que recebem pouco apoio de profissionais de saúde no período de reabilitação(19-20).

O momento de transição do cuidado da fase hospitalar para o contexto domiciliar ocorreu de modo solitário e os pacientes e seus familiares resolveram as demandas de cuidado de modo isolado, sem acompanhamento profissional ${ }^{(4,7,17,19)}$. Diante desse cenário, os pacientes e familiares carentes de informações ficam à mercê de ajuda de uma rede assistencial fragilizada e fragmentada e estão sob o risco de vivenciarem eventos adversos que poderão levar a complicações e a readmissões ao longo desse processo $^{(4,5,22-24)}$.

Os participantes não estavam satisfeitos com o acesso as intervenções para reabilitação após a alta hospitalar e mencionaram que tiveram restrições para a continuidade do cuidado no contexto comunitário. Esse aspecto também foi evidenciado em estudos conduzidos em países desenvolvidos que identificaram a fragmentação do cuidado, a falta de continuidade, de comunicação, de coordenação e de integração entre 
os serviços de saúde, a inexistência ou o difícil acesso ao serviço de reabilitação no contexto comunitário ${ }^{(23-}$ 24).

A efetiva reabilitação deve envolver uma avaliação biopsicossocial, com um conjunto de medidas interdisciplinares, visando capacitar a família para minimizar as chances de novos episódios ${ }^{(6-7,23)}$. O período de reabilitação tem sido negligenciado pelo sistema de saúde e as intervenções educativas, quando ocorrem, têm sido superficiais, sem considerar as necessidades específicas de cada pessoa e do seu núcleo familiar $^{(7,17,19,23)}$. Isso denota fragilidades no cumprimento dos princípios da universalidade e da equidade do sistema de saúde brasileiro, que traz prejuízos importantes para que essas pessoas tenham após o período de internação uma efetiva reinserção social e comunitária ${ }^{(6)}$.

Os pesquisadores descrevem que esse é um problema grave nos países desenvolvidos, e uma verdadeira tragédia, nos países em desenvolvimento, que enfrentam problemas relacionados a subutilização das infraestruturas e dos escassos recursos (humanos e materiais) em saúde ${ }^{(25)}$. Nesse sentido, a ineficiência no modo de produção do cuidado tende a resultar em atrasos no acompanhamento e seguimento do tratamento, dificuldades para referenciar as pessoas para os diferentes níveis de atenção a saúde, erros e outros eventos adversos indesejáveis que levam déficits para a resolutividade e a consequente decepção e insatisfação geral das pessoas, famílias e comunidades ${ }^{(25)}$.

\section{CONCLUSÕES}

As políticas públicas defendem um modelo baseado na prevenção e na promoção da saúde por meio da educação em saúde para a população, assistência multidisciplinar para intervenção no quadro clínico de AVC e reabilitação das disfunções, com suporte social formal às famílias, fortalecendo sua parceria no cuidado domiciliar.

Os achados mostraram uma dicotomia entre a efetiva empregabilidade das diretrizes das políticas públicas no processo de cuidado e reabilitação das pessoas que sobreviveram ao AVC e a realidade vivenciada por essas pessoas e seus familiares.

O que se tem observado é que a população ainda carece de conhecimento e sensibilização quanto aos fatores de risco e o impacto das disfunções relacionadas ao AVC sobre os aspectos físicos, sociais, financeiros e psicológicos, o que pode representar a negligência própria do cuidado com a saúde e a falta de apoio de outros profissionais, sobretudo o enfermeiro como gerente do cuidado integral.

A família é fundamental para o acesso aos serviços de saúde e garantia na manutenção da assistência, contudo a condução dessa assistência ocorre de maneira solitária. Portanto, as redes de atenção à saúde devem favorecer suporte aos familiares e explorar mais o seu potencial para o cuidado, assim como reorganizar o fluxograma de atendimento com a finalidade de garantir a efetiva ação dos serviços de saúde para atender a população adscrita.

A produção de resultados positivos do cuidado, após o AVC, requer que os sistemas de saúde estejam organizados de modo integrado e assegurem a continuidade do cuidado envolvendo intervenções centradas 
nas pessoas (indivíduo, família e comunidade), nos diferentes cenários de produção do cuidado, por meio da atuação integrada dos profissionais de saúde.

\section{AGRADECIMENTOS}

A Universidade do Estado da Bahia (UNEB), pelo apoio parcial para a realização do Programa de Pósgraduação Stricto sensu, Mestrado em Ciências Ambientais e Saúde, por meio da concessão da Bolsa do Programa de Apoio Capacitação de Docentes e Técnicos Administrativos (PAC-DT).

\section{FINANCIAMENTOS}

Esta pesquisa integra o projeto temático "O processo de reabilitação na perspectiva de idosos e familiares que vivenciaram o acidente vascular encefálico", financiado pela Fundação de Amparo a Pesquisa do Estado de Goiás aprovado no Edital Universal no 005/2012.

\section{REFERÊNCIAS}

1. Mukherjee D, Patil CG. Epidemiology and the global burden of stroke. World Neurosurg [Internet]. 2011 [acesso em: 31 Mar. 2016];76(6 Suppl):S85-90. Disponível em: http://dx.doi.org/10.1016/j.wneu.2011.07.023.

2. Zhang $\mathrm{Y}$, Chapman A, Plested M, Jackson D, Purroy F. The incidence, prevalence, and mortality of stroke in France, Germany, Italy, Spain, the UK, and the US: A literature review. Stroke Res Treat. [Internet]. 2012 [acesso em: 31 Mar. 2016];2012(2012):1-11. Disponível em: http://dx.doi.org/10.1155/2012/436125.

3. Lotufo PA, Benseñor IM. Stroke mortality in Brazil: one example of delayed epidemiological cardiovascular transition. Int J Stroke [Internet]. 2009 [acesso em: 31 Mar. 2016];4(1):40-1. Disponível em:

http://dx.doi.org/10.1111/j.1747-4949.2009.00240.x.

4. Fisher RJ, Gaynor C, Kerr M, Langhorne P, Anderson C, Bautz-Holter E, et al. A consensus on stroke: early supported discharge. Stroke [Internet]. 2011 [acesso em: 31 Mar. 2016];42(5):1392-7. Disponível em: http://dx.doi.org/10.1161/STROKEAHA.110.606285.

5. Gallacher K, Morrison D, Jani B, Macdonald S, May CR, Montori VM, et al. Uncovering treatment burden as a key concept for stroke care: a systematic review of qualitative research. PLoS Med [Internet]. 2013 [acesso em: 31 Mar. 2016];10(6):e1001473. Disponível em: http://dx.doi.org/10.1371/journal.pmed.1001473.

6. Lindsay P, Furie KL, Davis SM, Donnan GA, Norrving B. World Stroke Organization global stroke services guidelines and action plan. Int J Stroke [Internet]. 2014 [acesso em: 31 Mar. 2016];9 Suppl A100:4-13. Disponível em: http://dx.doi.org/10.1111/ijs.12371.

7. Chen L, Xiao LD, De Bellis A. First-time stroke survivors and caregivers' perceptions of being engaged in rehabilitation. J Adv Nurs [Internet]. 2016 [acesso em: 31 Mar. 2016];72(1):73-84. Disponível em:

http://dx.doi.org/10.1111/jan.12819.

8. Morse JM. Analytic strategies and sample size. Qual Health Res [Internet]. 2015 [acesso em: 31 Mar. 2016];25(10):1317-8. Disponível em: http://dx.doi.org/10.1177/1049732315602867.

9. Guest $\mathrm{G}$, Bunce A, Johnson L. How many interviews are enough? An experiment with data saturation and variability. Field methods [Internet]. 2006 [acesso em: 31 Mar. 2016];18(1):59-82. Disponível em: http://dx.doi.org/10.1177/1525822X05279903.

10. Braun V, Clarke V. Using thematic analysis in psychology. Qual Res Psychol [Internet]. 2006 [acesso em: 31 Mar. 2016];3(2):77-101. Disponível em: http://dx.doi.org/10.1191/1478088706qp063oa.

11. Polit DF, Beck CT. Nursing research: generating and assessing evidence for nursing practice. 9 o ed. Philadelphia: Wolters Kluwer/Lippincont Williams \& Wilkins; 2012.

12. Mackintosh JE, Murtagh MJ, Rodgers H, Thomson RG, Ford GA, White M. Why people do, or do not, immediately contact emergency medical services following the onset of acute stroke: qualitative interview study. PLoS One. 
[Internet]. 2012 [acesso em: 31 Mar. 2016];7(10):e46124. Disponível em: http://dx.doi.org/10.1371/journal.pone.0046124.

13. Salisbury L, Wilkie K, Bulley C, Shiels J. 'After the stroke': patients' and carers' experiences of healthcare after stroke in Scotland. Health Soc Care Community [Internet]. 2010 [acesso em: 31 Mar. 2016];18(4):424-32. Disponível em: http://dx.doi.org/10.1111/j.1365-2524.2010.00917.x.

14. Fernandes DR, Santos EA, Dias RS, Leite SRC, Sousa DES, Silva JS. Caracterização de pacientes com doença cerebrovascular: uma avaliação retrospectiva. Cad Pesqui [Internet]. 2013 [acesso em: 31 Mar. 2016];20(1):50-7. Disponível em: http://www.periodicoseletronicos.ufma.br/index.php/cadernosdepesquisa/article/view/1249. 15. Coole C, Radford K, Grant M, Terry J. Returning to work after stroke: perspectives of employer stakeholders, a qualitative study. J Occup Rehabil [Internet]. 2013 [acesso em: 31 Mar. 2016];23(3):406-18. Disponível em: http://dx.doi.org/10.1007/s10926-012-9401-1.

16. Daniel K, Wolfe CD, Busch MA, McKevitt C. What are the social consequences of stroke for working-aged adults? A systematic review. Stroke [Internet]. 2009 [acesso em: 31 Mar. 2016];40(6):e431-40. Disponível em:

http://dx.doi.org/10.1161/STROKEAHA.108.534487.

17. Simeone S, Savini S, Cohen MZ, Alvaro R, Vellone E. The experience of stroke survivors three months after being discharged home: A phenomenological investigation. Eur J Cardiovasc Nurs [Internet]. 2015 [acesso em: 31 Mar. 2016];14(2):162-9. Disponível em: http://dx.doi.org/10.1177/1474515114522886.

18. Bloom DE, Cafiero ET, Jané-Llopis E, Abrahams-Gessel S, Bloom LR, Fathima S, et al. The global economic burden of non-communicable diseases: report by the World Economic Forum and the Harvard School of Public Health [Internet]. Geneva: World Economic Forum; 2011 [acesso em: 31 Mar. 2016]. Disponível em:

http://apps.who.int/medicinedocs/documents/s18806en/s18806en.pdf.

19. Rodrigues RAP, Marques S, Kusumota L, Santos EB, Fhon JRS, Fabrício-Wehbe SCC. Transition of care for the elderly after cerebrovascular accidents - from hospital to the home. Rev Lat Am Enfermagem [Internet]. 2013 [acesso em: 31 Mar. 2016];21(spe):216-24. Disponível em: http://dx.doi.org/10.1590/S0104-11692013000700027.

20. Huang CY, Hsu MC, Hsu SP, Cheng PC, Lin SF, Chuang CH. Mediating roles of social support on poststroke depression and quality of life in patients with ischemic stroke. J Clin Nurs [Internet]. 2010 [acesso em: 31 Mar. 2016];19(19-20):2752-62. Disponível em: http://dx.doi.org/10.1111/j.1365-2702.2010.03327.x.

21. Marques AKMC, Landim FLP, Collares PM, Mesquita RB. Apoio social na experiência do familiar cuidador. Cien Saude Colet [Internet]. 2011 [acesso em: 31 Mar. 2016];16 Supl. 1:945-55. Disponível em:

http://dx.doi.org/10.1590/S1413-81232011000700026.

22. Ministério da Saúde. Diretrizes de atenção à reabilitação da pessoa com acidente vascular cerebral [Internet]. Brasília: Ministério da Saúde; 2013 [acesso em: 31 Mar. 2016]. Disponível em:

http://bvsms.saude.gov.br/bvs/publicacoes/diretrizes_atencao_reabilitacao_acidente_vascular_cerebral.pdf.

23. Teasell R, Meyer MJ, Foley N, Salter K, Willems D. Stroke rehabilitation in Canada: a work in progress. Top Stroke Rehabil [Internet]. 2009 [acesso em: 31 Mar. 2016];16(1):11-9. Disponível em: http://dx.doi.org/10.1310/tsr1601-11. 24. Maniva SJCF, Freitas CHA, Jorge MSB, Carvalho ZMF, Moreira TMM. Experiencing acute stroke: the meaning of the illness for hospitalized patients. Rev Esc Enferm USP [Internet]. 2013 [acesso em: 31 Mar. 2016];47(2):362-8.

Disponível em: http://dx.doi.org/10.1590/S0080-62342013000200013.

25. Nuño R, Coleman K, Bengoa R, Sauto R. Integrated care for chronic conditions: the contribution of the ICCC Framework. Health Policy [Internet]. 2012 [acesso em: 31 Mar. 2016];105(1):55-64. Disponível em:

http://dx.doi.org/10.1016/j.healthpol.2011.10.006. 